Original Article

\title{
Platelet Volume Indices as Non - Invasive Physiological Marker of Liver Fibrosis in Chronic Hepatitis C Patients
}

\author{
Yar Mohammad Nizamani, Aamir Hussain, Ahmed Hussain Suhag
}

ABSTRACT

BACKGROUND: Mean platelet volume (MPV), platelet distribution width (PDW) and P-LCR (platelet large cell ratio) are known platelet volume indices (PVI). Currently, the platelet volume indices (PVI) have emerged to determine its value as biomarker of liver fibrosis.

OBJECTIVES: The present study determined the Platelet volume indices (PVI) as non invasive physiological marker of liver fibrosis in chronic hepatitis $C$ patients.

STUDY DESIGN: Case control study

STUDY SETTINGS: Department of Gastroenterology, ISRA University Hospital and Asian Institute of Medical Sciences (AIMS) Hyderabad from May- December 2014.

SUBJECTS AND METHODS: A sample of 64 patients; 32 controls and 32 chronic hepatitis C cases were studied. Subjects were selected by non-probability (purposive) sampling as per inclusion and exclusion criteria. Roche Hitachi Hematolyzer was used for platelet count and platelet volume indices. Liver fibrosis was determined from liver biopsies by METAVIR Score. Data was analysed on Statistix 10.0 (USA) at $95 \%$ confidence interval $(P<0.05)$.

RESULTS: The PVI (MPV, PDW and P-LCR) and platelet count were raised in cases compared to controls $(p<0.05)$. PVI showed major differences for METAVIR SCORE $F_{0}-F_{4}$ of liver fibrosis $(p<0.05)$. MPV, PDW and P-LCR showed positive correlation $(r=0.93, r=0.80$ and $r=0.75)$ with METAVIR $(p<0.0001)$ respectively.

CONCLUSION: The Platelet volume indices may be used as non-invasive physiological markers for liver fibrosis in chronic hepatitis $\mathrm{C}$ patients instead of invasive technique of liver biopsy.

KEY WORDS: PVI, Chronic hepatitis C, Liver fibrosis, Metavir score.

This article may be cited as: Nizamani YM, Hussain A, Suhag AH. Platelet Volume Indices as Non - Invasive Physiological Marker of Liver Fibrosis in Chronic Hepatitis C Patients. J Liaquat Uni Med Health Sci. 2017;16(02):114-7. doi: 10.22442/jlumhs.171620517

\section{INTRODUCTION}

Hepatitis $\mathrm{C}$ virus (HCV) has become a major cause of chronic viral hepatitis (CHC), liver fibrosis, liver cirrhosis and liver malignancy throughout the World., World prevalence of $3 \%$ which accounts 170 million people has been reported by $\mathrm{WHO}^{1,2}$. Infectivity rate is estimated at 3-4 million people, of whom $65-80 \%$ are in chronic intermediate phase. Prevalence of liver cirrhosis and liver malignancy are estimated at $20 \%$ and $25 \%$ respectively. This accounts for high mortality and morbidity caused by $\mathrm{HCV}^{1,2}$.

Transmission of HCV occurs by sexual practices, blood transfusions, injections, tourists, barbers and shaving blades in the developed and developing countries. Unsafe injection practice and blood transfusion is the major mode of $\mathrm{HCV}$ transmission in Pakistan $^{3,4}$. Natural course of chronic hepatic C (CHC) culminates into hepatic fibrosis, and liver cirrhosis is its end stage. Grading of liver fibrosis is essential for the clinical staging of liver damage for the clinical management, for example for initiating the interferon therapy to cure the patient ${ }^{5-8}$. Currently, liver biopsy is used for histological scoring and is a gold standard, but it is highly invasive method. Various fibrosis scoring systems are available but Metavir is an ideal one. Recently, many non-invasive methods of liver fibrosis estimation for example elastography are under practice, but they need instruments and expert hands. Hence alternate methods of non-invasive nature are being searched for staging the liver fibrosis ${ }^{9}$.

$\mathrm{PVI}$ include; the mean platelet volume (MPV), platelet distribution width (PDW) and P-LCR (platelet large cell ratio) which are the determinants of platelet physiology ${ }^{10}$. A measure of platelet shape and size is known as the mean platelet volume (MPV). The platelet large cell ratio (P-LCR) is defined as the \% of large size platelets while platelet distribution width (PDW) shows the platelet distribution. Currently, the platelet volume indices (PVI) have emerged as highly researched topic as marker of liver fibrosis. Because PVI is nonexpensive easy to perform and valuable tool for the clinical diagnosis of liver fibrosis in $\mathrm{CHC}$ patients ${ }^{11-14}$. 
Keeping in view, the present study was conducted to determine Platelet volume indices in chronic hepatitis $C$ patients and their diagnostic utility for liver fibrosis in comparison to Metavir score at our tertiary care hospital.

\section{SUBJECTS AND METHODS}

The present case control study was conducted at the Department of Gastroenterology, ISRA University Hospital and Asian Institute of Medical Sciences (AIMS) Hyderabad, Sindh from May 2014 to December 2014. Prior permission was taken from the ethical review committee. Volunteer subjects were asked for written informed consent. A sample of 64 patients; 32 controls and 32 of chronic hepatitis C cases were studied. Subjects were selected by nonprobability (purposive) sampling as per inclusion and exclusion criteria. Male subjects of chronic hepatitis C without any associated co-morbid condition were the inclusion criteria. $\mathrm{CHC}$ with chronic hepatitis $\mathrm{B}(\mathrm{CHB})$, decompensated liver cirrhosis, esophageal varices, Variceal hemorrhage and bleeding disorders were the exclusion criteria.

Five $\mathrm{ml}$ of venous blood was collected by Venepuncture and processed on Roche Hitachi Hematolyzer platelet count and platelet volume indices. Percutaneous Liver biopsy of liver fibrosis patients was performed only. Procedure was carried out by a Gastroenterologist under Sonography guidance. Liver biopsy was stained with Hematoxylin and eosin. Liver fibrosis was graded by METAVIR score five categories as F0, F1, F2, F3 and F4.

Data was collected on a pre-designed pre-structured proforma. The confidentiality of study population was strictly maintained. The Data was analyzed using Statistix 10.0 (USA). One way analysis of variance (ANOVA) and student t-test were used for continuous variable data. Metavir intra- and inter- group comparisons was calculated by post-hoc Tukey-Cramer test. Pearson's correlation was used for the correlation estimation. Data was analyzed at $95 \%$ confidence interval $(P<0.05)$ taken statistically significant.

\section{RESULTS}

The PVI (MPV, PDW and P-LCR) and platelet count showed were raised in cases compared to controls $(p<0.05)$ (Table I). PVI showed major differences for METAVIR SCORE $F_{0}-F_{4}$ of liver fibrosis $(p<0.05)$. MPV, PDW and P-LCR showed positive correlation $(r=0.93, r=0.80$ and $r=0.75)$ with METAVIR ( $p$ $<0.0001$ ) respectively. MPV, PDW, and P-LCR among Metavir groups- $F_{0}$ through $F_{4}$ of liver fibrosis score showed highly significant differences $(p=0.0001)$ (Table II). A positive association was found between liver fibrosis score and MPV, PDW and P-LCR $(r=0.93, r=0.80$ and $r=0.75)(p<0.0001)$ respectively. Platelet counts showed non-significant correlation with Metavir score (Table III).

TABLE I: PLATELET VOLUME INDICES (PVI) IN CONTROLS AND CASES

\begin{tabular}{|l|l|l|l|}
\hline & \multicolumn{1}{|c|}{$\begin{array}{c}\text { Cases } \\
(\mathbf{n = 3 0 )}\end{array}$} & $\begin{array}{c}\text { Controls } \\
(\mathbf{n = 3 0 )}\end{array}$ & P-value \\
\hline MPV (fl) & $11.28 \pm 1.19$ & $9.23 \pm 0.55$ & 0.0001 \\
\hline PDW (\%) & $16.21 \pm 4.27$ & $10.37 \pm 0.43$ & 0.0001 \\
\hline P-LCR $(\%)$ & $36.4 \pm 2.93$ & $27.5 \pm 6.20$ & 0.0001 \\
\hline PC $\left(10^{6} / \mu \mathrm{L}\right)$ & $290.1 \pm 76.1$ & $302.5 \pm 67.1$ & 0.04 \\
\hline
\end{tabular}

MPV- mean platelet volume, PDW- platelet distribution width, P-LCR- platelet large cell ratio, PC- Platelet count. Student t-test

TABLE II: PLATELET VOLUME INDICES (PVI) ACCORDING TO METAVIR SCORE IN CASES $(n=30)$

\begin{tabular}{|l|l|l|l|l|}
\hline $\begin{array}{c}\text { METAVIR } \\
\text { score }\end{array}$ & \multicolumn{1}{|c|}{ MPV (fl) } & PDW (fI) & P - LCR (\%) & PC (x10 $/ \mu \mathrm{L})$ \\
\hline Score F0 & $9.90 \pm 0.17$ & $12.43 \pm 1.21$ & $33.49 \pm 2.15$ & $269.62 \pm 70.38$ \\
\hline Score F1 & $10.25 \pm 0.12$ & $13.68 \pm 2.15$ & $34.01 \pm 0.83$ & $269.81 \pm 65.53$ \\
\hline Score F2 & $10.81 \pm 0.19$ & $15.11 \pm 2.01$ & $37.15 \pm 2.90$ & $292.23 \pm 113.04$ \\
\hline Score F3 & $11.78 \pm 0.30$ & $17.45 \pm 1.15$ & $38.13 \pm 2.10$ & $319.56 \pm 118.09$ \\
\hline Score F4 & $13.19 \pm 0.60$ & $20.80 \pm 3.62$ & $39.27 \pm 1.45$ & $287.75 \pm 38.12$ \\
\hline P-value & 0.0001 & 0.0001 & 0.0001 & 0.081 \\
\hline
\end{tabular}

MPV- mean platelet volume, PDW- platelet distribution width, P-LCR- platelet large cell ratio, PC- Platelet count. ANOVA testing

TABLE III: CORRELATION CO-EFFICIENT OF PLATELET VOLUME INDICES AND LIVER FIBROSIS (METAVIR SCORE) IN CASES

\begin{tabular}{|l|l|l|l|l|}
\hline & MPV (fl) & PDW (fl) & P-LCR (\%) & PC (x10 $/$ dl) \\
\hline r-value & $0.921^{* *}$ & $0.812^{* *}$ & $0.735^{* *}$ & 0.104 \\
\hline$p$-value & 0.0001 & 0.0001 & 0.0001 & 0.426 \\
\hline
\end{tabular}

**. Correlation co-efficient ( $r$-value) is significant at the 0.01 level (2-tailed)

MPV- mean platelet volume, PDW- platelet distribution width, P-LCR-platelet large cell ratio, PC-Platelet count

\section{DISCUSSION}

The present research is being reported for the first time on an ever increasing health problem of $\mathrm{CHC}$. Non- invasive techniques are preferred over the invasive techniques like liver biopsy. The cases for this study were taken from the Department of Gastroenterology, ISRA University and Asian Institute of Medical Sciences (AIMS), Hyderabad. The Department of Gastroenterology of Isra University caters thousands $\mathrm{CHC}$ patients yearly. The present study 
was conducted to determine the Platelet volume indices (PVI) in comparison to Metavir score of liver fibrosis. As the $\mathrm{CHC}$ infection runs a stealthily natural course, hence there is need of some non-invasive technique for screening and predicting the onset of liver fibrosis ${ }^{13-15}$. Platelet volume indices $(\mathrm{PVI})$ are negative correlates of platelet size and physiology. Physiologically, the circulating platelets are closely regulated within limits by the bone marrow feedback mechanisms. In cases of increased platelet consumption, megakaryocyte proliferate, newly released platelets are large in number and more aggressive physiologically. Megakaryocyte proliferation is influenced by the thrombopoietin ${ }^{13,15}$. As the liver fibrosis is associated with clotting and hemostasis abnormalities, hence there is a change in the circulating platelets, majority of who are newly released from bone marrow with different PVI. This concept was exploited for the present study.

The present study reports raised PVI in $\mathrm{CHC}$ patients compared to the controls. The PVI were compared with Metavir score and a positive correlation was found. This finding is of clinical importance as it may be used as a non-invasive physiological marker of liver fibrosis. PVI shows positive correlation with the METAVIR scores $F_{0}-F_{4}$ and is found statistically significant $(p<0.05)$ in the present study reports (table III). The findings of raised MPV of present study is a worth finding as it is consistent with a previously reported study ${ }^{16}$. A previous study ${ }^{17}$ reported the MPV and PDW were found increased in $\mathrm{CHC}$ fibrosis compared to non-fibrosis patients. These findings of present study are in agreement with previous study ${ }^{17}$. Above previous study reported the PDW was found highly indicative of advanced liver fibrosis, hence our present study supported by above study. Chen B, et $\mathrm{al}^{18}$ reported the raised $\mathrm{PVI}$ values in the liver fibrosis of chronic hepatitis $B$. They reported positive correlation with Metavir scoring, which is consistent finding with the present study. Many previous studies ${ }^{6,19-21}$ have reported PVI results which support our present study and previous studies ${ }^{17,18}$. The finding of positive correlation of severity of liver fibrosis (Metavir score) of present study is supported by previous studies ${ }^{21,22}$ as they reported positive association of severity of liver fibrosis with PVI. The findings of raised MPV, PDW, P-LCR and reduced PC of present study are supported by previous medical research studies as mentioned above. The present study reports the PVI indices may prove helpful as non-invasive physiological marker of liver fibrosis in chronic hepatic C patients. The present study concludes sufficient information has been achieved on the topic which needs further large sample nationwide studies to make cut off values and guidelines on the PVI as marker of liver fibrosis. The major limitation of present study is a small sample size, however, PVI were compared with gold standard test; the liver biopsy which is a strong evidence of the association.

\section{CONCLUSION}

The Platelet volume indice (PVI) were elevated in chronic hepatitis C patients. The PVI are easy, inexpensive, and non-invasive markers which may be exploited for predicting liver fibrosis at earlier stage even in remote rural areas of country, but this needs further large scale nationwide studies.

\section{REFRENCES}

1. Bhatti S, Manzoor S. Molecular epidemiology and clinical features of Hepatitis C Virus (HCV) in epidemic areas of Interior Sindh, Pakistan. Pak J Med Sci 2016; 32(5):1279-82.

2. Umer $M$, lqbal $M$. Hepatitis $C$ virus prevalence and genotype distribution in Pakistan: Comprehensive review of recent data. World $J$ Gastroenterol 2016; 22(4): 1684-1700.

3. Qureshi H, Bile KM, Jooma R, Alam SE, Afridi HU. Prevalence of hepatitis $B$ and $C$ viral infections in Pakistan: findings of a national survey appealing for effective prevention and control measures. East Mediterr Health J 2010; 16 Suppl: S15-23.

4. Altaf A, Janjua NZ, Hutin Y. The cost of unsafe injections in Pakistan and challenges for prevention program. J Coll Physicians Surg Pak 2007; 16: $622-4$.

5. Maruyama H, Shiha G, Yokosuka O, et al. Non-invasive assessment of portal hypertension and liver fibrosis using contrast-enhanced Ultrasonography. Hepatol Int 2016; 10(2):267-76.

6. Li SM, Li GX, Fu DM, et al. Liver fibrosis evaluation by ARFI and APRI in chronic hepatitis C. World J Gastroenterol 2014; 20(28):9528-33.

7. Castera L. Non-invasive assessment of liver fibrosis in chronic hepatitis C. Hepatol Int 2011; 5 (2): 625-34.

8. Sarin SK, Kumar A, Aalmeida JA, et al. Acute-on -chronic Liver failure: consensus recommendations of the Asian Pacific Association for the study of Liver (APASL). Hepatol Int 2009; 3 (1): 269-82.

9. Xiao G, Yang J, Yan L. Comparison of diagnostic accuracy of aspartate aminotransferase to platelet ratio index and fibrosis-4 index for detecting liver fibrosis in adult patients with chronic hepatitis B virus infection: a systemic review and meta-analysis. Hepatology 2015; 61(1): 292-302.

10. Buttarello M, Plebani M. Automated Blood Cell Counts: State of the Art. American J Clin Pathol 2008; 130(1): 104-16. 
11. Ucar F, Sezer S, Ginis Z, et al. APRI, the FIB-4 score, and Forn's index have noninvasive diagnostic value for liver fibrosis in patients with chronic hepatitis B. Eur J Gastroenterol Hepatol 2013; 25(9): 1076-81.

12. Ullah F, Khan S, Afridi AK, Ur Rehman S. Frequency of different causes of cirrhosis liver in local population. Gomal J Med Sci 2012; 10 (2):178-81.

13. Castera L. Noninvasive methods to assess liver disease in patients with hepatitis B or C. Gastroenterology 2012; 142(6):1293-1302.e4.

14. Zeng DW, Zhang JM, Liu YR, et al. A new model for predicting liver cirrhosis in chronic hepatitis $B$ virus carriers with low serum alanine transaminase activity. Clin Res Hepatol Gastroenterol 2014; 38(6):727-34.

15. Zubcevic N, Mesihovic R, Zubcevic S. Usefulness of laboratory data in estimation of Crohn's disease activity. Med Arh 2010; 64(1):33-6.

16. Karaman $H$, Karakukcu $C$, Karaman $A$, et al. Mean platelet volume as a fibrosis marker in patients with chronic hepatitis C. Turk J Med Sci 2013; 43(1):39-45.

17. Zeng DW, Dong J, Liu YR, Jiang JJ, Zhu YY. Noninvasive models for assessment of liver fibrosis in patients with chronic hepatitis B virus infection Wosrld J Gastroenterol 2016; 22(29): 6663-72.

18. Chen B, Ye B, Zhang J, Ying L, Chen Y. RDW to Platelet Ratio : A novel noninvasive index for predicting hepatic fibrosis and cirrhosis in chronic hepatitis B. PLos One 2013;8(7):e68780.

19. Park HS, Kim YJ, Yu MH, et al. Three Tesla magnetic resonance elastography for hepatic fibrosis: comparison with diffusion-weighted imaging and gadoxetic acid enhanced magnetic resonance imaging. World J Gastroenterol 2014; 20(46):17558-7.

20. Tseng PL, Wang JH, Hung $\mathrm{CH}$, et al. Comparisons of noninvasive indices based on daily practice parameters for predicting liver cirrhosis in chronic hepatitis $B$ and hepatitis $C$ patients in hospital and community populations. Kaohsiung $\mathrm{J}$ Med Sci 2013; 29(7):385-95.

21. Yan-Jun Z. Clinical significance of the variation of platelet parameters in patients with liver cirrhosis. J Xinxiang Med Coll 2011; 02: 575.

22. Purnak T, Olmez $S$, Torun $S$, et al. Mean platelet volume is increased in chronic hepatitis $\mathrm{C}$ patients with advanced fibrosis. Clin Res Hepatol Gastroenterol 2013; 37(1):41-6.

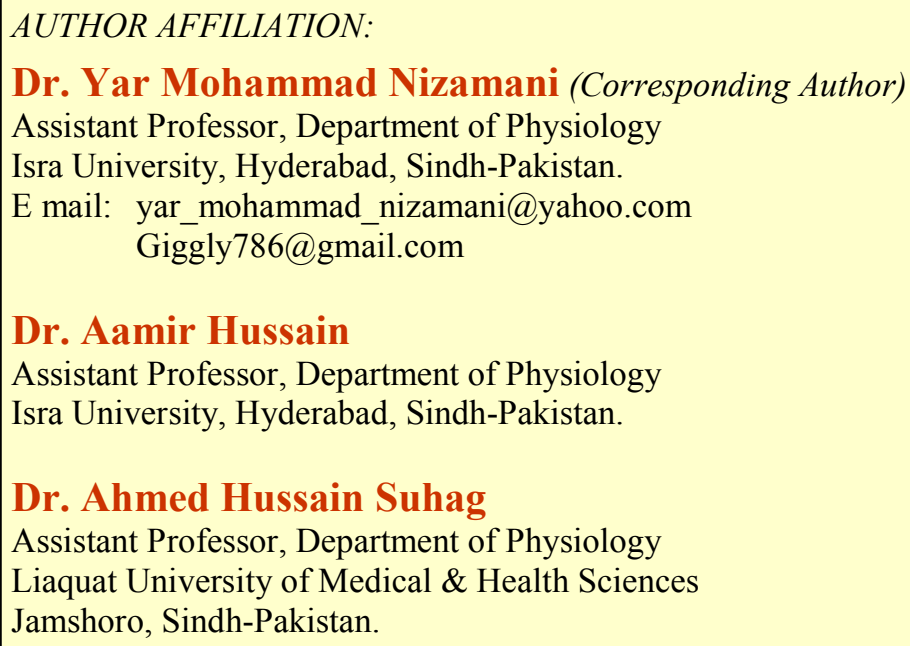

